\section{Borderline Personality Disorder in Individuals with Intellectual Disability}

\section{Abstract}

Both Intellectual Disability (ID) and Borderline Personality Disorder (BPD) can be associated with behavioral dysregulation including self-harm, impulsivity, and intense anger. When these two entities overlap, clinicians are faced with limited guidance. The American Psychiatric Association has guidelines for the diagnosis and treatment of Borderline Personality Disorder as well as the diagnosis of Intellectual Disability. The NADD press has published an adaptation of these diagnostic criteria for Borderline Personality Disorder as they apply to those with ID, but there are not yet any guidelines for treatment in co-occurrence of these disorders. This paper will review the literature on the co-occurrence disorders and propose treatment recommendations following the existing literature and in adapting current treatment strategies for people with ID.

Keywords: Borderline personality disorder; Intellectual disability; Adapted treatment for co-occurring BPD and ID

\author{
Allison Cowan* \\ Wright State University, Boonshoft \\ School of Medicine, 627 S. Edwin C. \\ Moses Blvd, Dayton, Dayton, $\mathrm{OH}$, United \\ States
}

\section{*Corresponding author: Allison Cowan}

\section{झ allison.cowan@wright.edu}

Assistant Professor, Psychiatry, Wright State University, Boonshoft School of Medicine, Dayton, $\mathrm{OH} 45417$, United States.

Citation: Cowan A (2018) Borderline Personality Disorder in Individuals with Intellectual Disability. J Child Dev Disord. Vol.4 No.2:6

\section{Introduction}

Borderline personality disorder (BPD) is described in the DSM-5 as "a pervasive pattern of instability of interpersonal relationships, self-images, and affects" [1]. The prevalence of BPD is estimated to be close to $6 \%$ in the United States [2], and the prevalence of intellectual disability is estimated to be about $1 \%$ worldwide $[3,4]$. The literature about the co-occurrence of these two disorders is scarce. While not a representative sample, Lindsay et al. found a prevalence of $39.9 \%$ of any personality disorder in forensic intellectual disability services and Borderline Personality Disorder being $10-13 \%$ [5]. There remains significant variance in the understood prevalence of the disorder due to diagnosis being controversial in regard to diagnostic overshadowing [6]. Symptoms of BPD in this case would be attributed to the individual's intellectual disability rather than to a diagnosable disorder. Some authors advise caution given that people with ID already have one devalued disorder and that to add another devalued disorder could potentially be even more stigmatizing [7]. The Standardized Assessment of Personality is a semi-structured diagnostic interview which relies on having a collateral historian who has known the patient well for at least five years [8]. It has been shown to have validity for the diagnosis of personality disorder in adults with learning disability and severe behavioral problems [9]. However, there has not been convincing research into the true prevalence of the co-occurrence of these two disorders.

\section{Diagnosis of BPD in Individuals with ID}

Individuals with ID are often brought to the attention of a psychiatrist for aggression or "challenging behavior." This is not unlike BPD with self-injury, parasuidical behavior, suicidal ideation, and suicide attempts. Also, like individuals with ID, individuals with BPD also have neuropsychological deficits including lower verbal, performance, and full-scale IQ scores compared to controls [10]. They were also found to have impaired motor skills visuomotor integration and with a susceptibility to interference [10]. Findings of deficits in memory, executive functioning, and processing speed by Unoka and Richman [11] have been shown to be mediated by level on education, which suggests that people with intellectual disabilities and BPD would fare worse. Negative affects appear to interfere with cognitive processing in people with BPD in a way that is different from controls, and decreased activation in the orbitofrontal cortex [12]. The implications of these deficits in BPD are not yet elucidated in individuals with co-occurring ID and BPD, but the overlap of these disorders could indicate a poorer prognosis.

DM-ID-2 describes the following limitations in diagnosing individuals with intellectual disabilities with personality disorders [13]. These include

- Any diagnosis of personality disorder should take into account personal characteristics in the context of a normal cultural framework... 
- The IDD itself is likely to present some features that are the same as those of personality disorder...

- The IDD is likely to have contributed to delayed development and a degree of immaturity.

- Institutional experience is pervasive among this client group, and adaptation to institutional life should be taken into account when considering a diagnosis.

- Many individuals with IDD have experienced a protected upbringing, giving them a reduced access to opportunities to learn social norms, community skills, and so on...

- Along with other authors, the current committee recommends the use of behavioral observation and informant information, collected in a standardized manner, as a primary information source in making a diagnosis.

- DM-ID-2, 2016.

Diagnosis in individuals with ID can be difficult in general. Verbal and nonverbal communication can be impacted. In working with people with ID, more information about specifics like times, dates, and patterns of behavior might be gathered from collateral sources such as caregivers and family since a person with ID might not know these types of information. When addressing the diagnosis of BPD in individuals with IDD, individuals with ID are "generally more reliant on caregivers than are other sections of the population" [13]. For example, if the criteria for avoiding real or imagined abandonment is to be met, care must be taken to remember that individuals with ID have real reason to fear if a primary, relied-upon caregiver is not present. This person may be responsible for transportation to work, helping with ADLs, or cooking meals. The absence of this person may constitute a rea emergency, and not simply the threat of emotional abandonment. As noted by Alexander and Cooray [14], the diagnosis of personality disorders often requires subtle and subjective information like thoughts and feelings which can be difficult to obtain in people with receptive and expressive language barriers as well as cognitive limitations.

Of the nine criteria for diagnosis of Borderline Personality, eliciting the criteria of identity disturbance and chronic emptiness can be thought of as one of the most challenging as the skills required for the understanding of these concepts are "fairly sophisticated" [13]. Additionally, making diagnosis more difficult is the interrogative suggestibility of those people with ID and the increased susceptibility to leading questions [15]. This is often called "yessing" the interviewer-the individual answers questions with yes instead of admitting ignorance or providing an answer the individual predicts will upset the examiner. There remains the exhortation that self-injury and anger dysregulation are often found in people with intellectual disability and that other causes be discounted before making a diagnosis of BPD [13]. Otherwise, the DM-ID-2 leaves fairly unchanged the nine criteria for the diagnosis of BPD in individuals with ID.

\section{Treatment of BPD in Individuals with ID}

The American Psychiatric Association encourages a thorough assessment of suicide risk factors and reminds clinicians that the primary treatment for BPD is psychotherapy, complemented by symptom-targeted pharmacotherapy (APA, 2010). While professionals evaluate the treatment of patients with personality disorder and mild ID will take longer and that longstanding reduction in behavioral problems is less likely [16], it has been demonstrated that psychotherapy adapted for individuals with ID is effective $[17,18]$. Additionally, there is evidence that treatment can be effective for co-occurring BPD and ID [19-21]. Dialectical Behavioral Therapy (DBT) has evidence of reducing level of risks and improvement in overall functioning in individuals with ID and BPD [20]. fMRI has shown that DBT reduces amygdalar hyperactivity in individuals with BPD alone [22]. Roscoe et al. found that while participants showed varying levels of understanding of DBT, there were themes of finding DBT as helpful and beneficial [23]. Large reductions in challenging behaviors in individuals with ID and BPD were shown in a four-year study with DBT and a Skills System [24].

Good Psychiatric Management of Borderline Personality Disorder is described as once weekly individual therapy (if useful), case management, and prudent medication management if necessary [25]. GPM has been shown to be as effective as DBT for individuals without ID across a broad range of outcome measures [26,27]. The method proposed here is to adapt the core tenets of GPM to treatment of individuals with co-occurring ID and BPD. The basic principles of GPM are

- Offer psychoeducation

- Be active, not reactive

- Be thoughtful

- The relationship is real as well as professional

- Convey that change is expected

- Foster accountability

- Maintain a focus on life outside of treatment

- Be flexible, pragmatic, and eclectic

- Good Psychiatric Management for BPD, 2014

The psychoeducation provided in GPM is that the majority of people diagnosed with BPD will no longer meet criteria for BPD at ten years $[28,29]$. Another aspect of psychoeducation is that genetic factors have a significant relationship in developing the disorder as supported by family and twin studies $[30,31]$. This is in opposition to previous stance that patients with BPD are "untreatable" and that the failures in treatment "were explained solely by the borderline patient's pernicious motivations" [32]. GPM also calls for the frank diagnosis of BPD, walking through the criteria with patients. In individuals with ID and BPD, these conversations often provide profound relief. Knowledge of an illness and treatment results in reduced anxiety [33]. Families and caregivers are comforted to have a diagnosis that fits with their loved one's symptoms. This can function to reduce shame and to increase acceptance and support and reinforcing that the symptoms that the illness is not the fault of the patient or the family of origin. The adaptation of psychoeducation would be explaining terminology in clear terminology.

The tenets of "be active, not reactive"; "be thoughtful"; and "be flexible, pragmatic, and eclectic" are a function of therapeutic 
stance [25]. Rather than the caricature of a silent psychoanalyst, clinicians working with individuals with BPD are encouraged to be warm, empathic, and curious as well as using techniques that can be more supportive or more interpretative. This naturally leads to the principle that the relationship is a real relationshipthe clinician should "acknowledge...mistakes" and "accept idealization, but do not encourage unrealistic expectations" [25]. These should also all hold true for working with individuals with ID. Clinicians may have an easier time forming a therapeutic alliance with the caregiver who brings the patient, but the primary relationship should be with the individual being treated.

The remaining principles focus on life outside the treatment office. "Change is expected," "accountability," and "focus on life outside treatment" all stress the importance of making improvements within therapy and that the individual participate in social rehabilitation. The reliance of individual with BPD on romantic relationships for feelings of connectedness and worth can be problematic, but GPM encourages patients to focus on work-either volunteer work or paid work. These GPM principles are easily translated to work with people with ID and BPD. There are many services to encourage individuals with ID to find supported or individual employment, either through sheltered workshops or through boards or departments of developmental disabilities. Interestingly, attending a training for GPM has the additional effect of decreasing dislike of patients with BPD and decreasing the belief that BPD's prognosis is hopeless as well as increasing the belief that effective psychotherapies exist [34]. Conveying that change is likely and expected fits well with the evidence of likely remission for a significant portion of people with BPD. However, there remains a need for long-term followup studies for people with co-occurring ID and BPD.

Particular attention should be paid to interpersonal hypersensitivity-the reaction to a real or perceived slight or insult with emotional instability [35]. Caregivers can present to psychiatric offices with reports of "he/she just got enraged out of nowhere." When looking at the specifics of the event, often a perceived loss or slight can be determined as the inciting event. Educating the interdisciplinary team about these events precipitating a response seemingly out of proportion can be helpful in understanding the individual with ID and BPD as well as generating increased empathy.

While psychotherapies are the preferred treatment in working with people with BPD, polypharmacy occurs with most patients with BPD [36-38]. Polypharmacy also occurs at higher rates in individuals with ID than the general population. A 2016 study by Deb et al. showed that $89 \%$ of patients with challenging behavior were receiving psychotropic medications and $45 \%$ received more than on psychotropic medication. One study found that individuals with ID were more likely to live at home, more likely to have been prescribed CNS medications excluding benzodiazepines and mood stabilizers that are also anticonvulsants, and more likely to have had psychiatric hospitalization [39]. There is no medication that has received the Food and Drug Administration indication for the treatment of BPD, but there is some evidence that medications may be helpful when targeted to a specific symptom [40]. There is insufficient evidence, however, to support the use of psychotropic medications for the challenging behaviors described in intellectual disabilities [41]. If medications are used for people with ID and BPD, functional analyses can be used to measure the effects of psychotropic medications and medication changes [42]. The International guide to prescribing psychotropic medications to individuals with ID recommends the lowest optimal dose, regular follow-up and consideration of discontinuation of treatment [43]. This is also what is recommended in GPM in considering use of psychotropic medications to treat symptoms of BPD [25].

The recent focus on Trauma-Informed care in ID has had the impact of educating direct care staff about the existence and sequelae of trauma [44]. Rates of mistreatment of people with ID have been found to be higher than that of control groups [45], and individuals with BPD report higher rates of childhood maltreatment [46]. Taking these factors into consideration would be beneficial for families and caregivers. Other treatments such as EMDR and Horticultural Therapy have also been shown to be helpful in individuals with ID $[47,48]$.

\section{Clinical Vignette}

Ms. A is a 28-year-old woman with a diagnosis of Mild Intellectual Disability. She presents with her mother for anger outbursts resulting in property destruction and physical violence. Her workshop staff send in written incident reports that state these episodes "come out of nowhere." When discussing the latest event in more detail with her therapist, Ms. A reported that her favorite staff person was going to go on the scheduled outing on another van and Ms. A felt left out. Ms. A reported, "She likes those other people better than she likes me." When gathering history, Ms. A also endorses chaotic, intense relationships characterized by dating one person for a week, typically becoming mad at him, breaking up and immediately starting to date someone else. She endorses affective instability in response to interpersonal hypersensitivity, e.g. is elated with positive feedback from supervisor but enraged when roommate didn't hear her request from the other room and assumed she was ignoring her. Ms. A also reports binge eating and spending her clothes and necessities allowance on items that she does not need, later regretting it. When upset after a fight with her roommate, Ms. A threatened to kill herself. When she was calmed, she reported that she did not mean it, but that she was only upset. This also follows a pattern that when Ms. A is upset, she threatens suicide but is typically redirected.

Ms. A's treatment team made the diagnosis of Borderline Personality Disorder. Her family reported that she had previously been diagnosed with Bipolar Disorder but that when described, those symptoms did not fit the BPD criteria did. Her mother reported, "It's like that list of borderline symptoms was written about her." Ms. A's team worked with her on connecting her feelings and reactions to events of interpersonal hypersensitivity and using coping skills rather than property destruction. She was able to get a volunteering job working with animals at a shelter and reported that it made her feel good to help. Her psychiatrist was able to slowly reduce some of the medications that she was taking. Ms. A is doing better at two-year follow-up. 


\section{Conclusion}

While there remains lack of research about the diagnosis of BPD in individuals with ID, the adaptation of DSM- 5 criteria by in the DM-ID-2 provides guidance about the potential pitfalls and intricacies of working with people with ID. There are several

\section{References}

1 American Psychiatric Association (2013) Diagnostic and statistical manual of mental disorders (DSM- $5^{\circledR}$ ). American Psychiatric Pub.

2 Grant BF, Chou SP, Goldstein RB, Huang B, Stinson FS, et al. (2008) Prevalence, correlates, disability, and comorbidity of DSM-IV borderline personality disorder: results from the Wave 2 National Epidemiologic Survey on Alcohol and Related Conditions. J Clin Psychiatry 69: 533-545.

3 Maulik PK, Mascarenhas MN, Mathers CD, Dua T, Saxena S (2011) Prevalence of intellectual disability: a meta-analysis of populationbased studies. Res Dev Disabil 32: 419-436.

4 Westerinen H, Kaski M, Virta L, Kautiainen H, Pitkälä KH, et al. (2017) The nationwide register-based prevalence of intellectual disability during childhood and adolescence. J Intellect Disabil Res 61: 802-809.

5 Lindsay WR, Hogue T, Taylor JL, Mooney P, Steptoe L, et al. (2006) Two studies on the prevalence and validity of personality disorder in three forensic intellectual disability samples. J Forens Psychiatry Psychol 17: 485-506.

6 Moreland J, Hendy S, Brown F (2008) The validity of a personality disorder diagnosis for people with an intellectual disability. J Appl Res Intellect Disabil 21: 219-226.

7 Lindsay WR, Steptoe L, Hogue TE, Taylor JL, Mooney P, et al. (2007) Internal consistency and factor structure of personality disorders in a forensic intellectual disability sample. J Intellect Dev Disabil 32: 134-142.

8 Pilgrim J, Mann A (1990) Use of the ICD-10 version of the Standardized Assessment of Personality to determine the prevalence of personality disorder in psychiatric in-patients. Psychol Med 20: 985-992.

9 Flynn A, Matthews H, Hollins S (2002) Validity of the diagnosis of personality disorder in adults with learning disability and severe behavioural problems: preliminary study. Br J Psychiatry 180: 543-546.

10 Swirsky-Sacchetti T, Gorton G, Samuel S, Sobel R, Genetta-Wadley A, et al. (1993) Neuropsychological function in borderline personality disorder. J Clin Psychol 49: 385-396.

11 Richman MJ, Unoka Z (2015) Mental state decoding impairment in major depression and borderline personality disorder: metaanalysis. Br J Psychiatry 207: 483-489.

12 Soloff PH, White R, Omari A, Ramaseshan K, Diwadkar VA (2015) Affective context interferes with brain responses during cognitive processing in borderline personality disorder: fMRI evidence. Psychiatry Res 233: 23-35.

13 Fletcher RJ, Barnhill J, Cooper SA (2016) Diagnostic manualintellectual disability (DM-ID-2): a textbook of diagnosis of mental disorders in persons with intellectual disability. National Association for the Dually Diagnosed Press.

14 Alexander R, Cooray S (2003) Diagnosis of personality disorders in learning disability. Br J Psychiatry 182: s28-s31. therapies that have evidence of being effective in treating individuals with ID including DBT and CBT. This article suggests adapting Good Psychiatric Management for BPD for treatment of individuals with ID. There remains a great need for further research both in the diagnosis and optimal treatment of people with ID and BPD.

15 Clare ICH, Gudjonsson GH (1993) Interrogative suggestibility, confabulation, and acquiescence in people with mild learning disabilities (mental handicap): implications for reliability during police interrogations. Br J Clin Psychol 32: 295-301.

16 van den Hazel T, Didden R, Korzilius H (2009) Effects of personality disorder and other variables on professionals' evaluation of treatment features in individuals with mild intellectual disabilities and severe behavior problems. Res Dev Disabil 30: 547-557.

17 Macdonald J, Sinason V, Hollins S (2003) An interview study of people with learning disabilities' experience of, and satisfaction with, group analytic therapy. Psychol Psychother 76: 433-453.

18 Brown M, Duff H, Karatzias T, Horsburgh D (2011) A review of the literature relating to psychological interventions and people with intellectual disabilities: issues for research, policy, education and clinical practice. J Intellect Disabil 15: 31-45.

19 McNair L, Woodrow C, Hare D (2017) Dialectical behaviour therapy [DBT] with people with intellectual disabilities: a systematic review and narrative analysis. J Appl Res Intellect Disabil 30: 787-804.

20 Sakdalan JA, Shaw J, Collier V (2010) Staying in the here-and-now: a pilot study on the use of dialectical behaviour therapy group skills training for forensic clients with intellectual disability. J Intellect Disabil Res 54: 568-572.

21 Mevissen L, De Jongh A (2010) PTSD and its treatment in people with intellectual disabilities: a review of the literature. Clin Psychol Rev 30: 308-316.

22 Goodman M, Carpenter D, Tang CY, Goldstein KE, Avedon J, et al. (2014) Dialectical behavior therapy alters emotion regulation and amygdala activity in patients with borderline personality disorder. J Psychiatr Res 57: 108-116.

23 Roscoe P, Petalas M, Hastings R, Thomas C (2016) Dialectical behaviour therapy in an inpatient unit for women with a learning disability: Service users' perspectives. J Intellect Disabil 20: 263-280.

24 Brown JF, Brown MZ, Dibiasio P (2013) Treating individuals with intellectual disabilities and challenging behaviors with adapted dialectical behavior therapy. J Ment Health Res Intellect Disabil 6: 280-303.

25 Gunderson JG, Links PS (2014) Handbook of good psychiatric management for borderline personality disorder. Arlington, VA, US: American Psychiatric Publishing, Inc.

26 McMain SF, Links PS, Gnam WH, Guimond T, Cardish RJ, et al. (2009) A randomized trial of dialectical behavior therapy versus general psychiatric management for borderline personality disorder. Am J Psychiatry 166: 1365-1374.

27 McMain SF, Guimond T, Streiner DL, Cardish RJ, Links PS (2012) Dialectical behavior therapy compared with general psychiatric management for borderline personality disorder: clinical outcomes and functioning over a 2-year follow-up. Am J Psychiatry 169: 650-661. 
28 Gunderson JG, Stout RL, McGlashan TH, Shea MT, Morey LC, et al. (2011) Ten-year course of borderline personality disorder: psychopathology and function from the collaborative longitudinal personality disorders study. Arch Gen Psychiatry 68: 827-837.

29 Zanarini MC, Frankenburg FR, Reich DB, Fitzmaurice G (2012) Attainment and stability of sustained symptomatic remission and recovery among patients with borderline personality disorder and axis II comparison subjects: a 16-year prospective follow-up study. Am J Psychiatry 169: 476-483.

30 Amad A, Ramoz N, Thomas P, Jardri R, Gorwood P (2014) Genetics of borderline personality disorder: systematic review and proposal of an integrative model. Neurosci Biobehav Rev 40: 6-19.

31 Gunderson JG (2011) Borderline personality disorder. N Engl J Med 364: 2037-2042.

32 Gunderson JG (2009) Borderline personality disorder: ontogeny of a diagnosis. Am J Psychiatry 166: 530-539.

33 Kiyohara LY, Kayano LK, Oliveira LM, Yamamoto MU, Inagaki MM, et al. (2004) Surgery information reduces anxiety in the pre-operative period. Rev Hosp Clin Fac Med Sao Paulo 59: 51-56.

34 Keuroghlian AS, Palmer BA, Choi-Kain LW, Borba CP, Links PS, et al. (2016) The effect of attending good psychiatric management (GPM) workshops on attitudes toward patients with borderline personality disorder. J Pers Disord 30: 567-576.

35 Gunderson JG, Lyons-Ruth K (2008) BPD's interpersonal hypersensitivity phenotype: a gene-environment-developmental model. J Pers Disord 22: 22-41.

36 Martín-Blanco A, Ancochea A, Soler J, Elices M, Carmona C, et al. (2017) Changes over the last 15 years in the psychopharmacological management of persons with borderline personality disorder. Acta Psychiatr Scand 136: 323-331.

37 Zanarini MC, Frankenburg FR, Hennen J, Silk KR (2004) Mental health service utilization by borderline personality disorder patients and Axis II comparison subjects followed prospectively for 6 years. J Clin Psychiatry 65: 28-36.
38 Deb S, Unwin G, Deb T (2015) Characteristics and the trajectory of psychotropic medication use in general and antipsychotics in particular among adults with an intellectual disability who exhibit aggressive behaviour. J Intellectual Disability Res 59: 11-25.

39 Lidher J, Martin DM, Jayaprakash MS, Roy A (2005) Personality disorders in people with learning disabilities: follow-up of a community survey. J Intellect Disabil Res 49: 845-851.

40 Lieb K, Völlm B, Rücker G, Timmer A, Stoffers JM (2010) Pharmacotherapy for borderline personality disorder: Cochrane systematic review of randomised trials. Br J Psychiatry 196: 4-12.

41 Trollor JN, Salomon C, Franklin C (2016) Prescribing psychotropic drugs to adults with an intellectual disability. Aust Prescr 39: 126-130.

42 Valdovinos MG, Henninger-McMahon M, Schieber E, Beard L, Conley B, et al. (2016) Assessing the impact of psychotropic medication changes on challenging behavior of individuals with intellectual disabilities. Int J Dev Disabil 62: 200-211.

43 Deb S, Kwok H, Bertelli M, Salvador-Carulla L, Bradley E, et al. (2009) International guide to prescribing psychotropic medication for the management of problem behaviours in adults with intellectual disabilities. World Psychiatry 8: 181-186.

44 https://www.samhsa.gov/nctic/trauma-interventions

45 Horner-Johnson W, Drum CE (2006) Prevalence of maltreatment of people with intellectual disabilities: a review of recently published research. Ment Retard Dev Disabil Res Rev 12: 57-69.

46 Battle CL, Shea MT, Johnson DM, Yen S, Zlotnick C, et al. (2004) Childhood maltreatment associated with adult personality disorders: findings from the collaborative longitudinal personality disorders study. J Pers Disord 18: 193-211.

47 Christie M, Thompson M, Miller PK, Cole F (2016) Personality disorder and intellectual disability: the impacts of horticultural therapy within a medium-secure unit. J Ther Hort 26: 3-17.

48 Mevissen L, Lievegoed R, De Jongh A (2011) EMDR treatment in people with mild ID and PTSD: 4 cases. Psychiatr Q 82: 43-57. 